\title{
Dropped Head Syndrome Caused by Immune-mediated Necrotizing Myopathy
}

Tatsuya Ueno ${ }^{1}$, Chieko Suzuki ${ }^{1}$, Ichizo Nishino ${ }^{2}$ and Masahiko Tomiyama ${ }^{1}$

Key words: dropped head syndrome, immune-mediated necrotizing myopathy, anti-SRP antibodies,

HyperCKemia, idiopathic inflammatory myopathies

(Intern Med 58: 3343-3344, 2019)

(DOI: 10.2169/internalmedicine.2930-19)

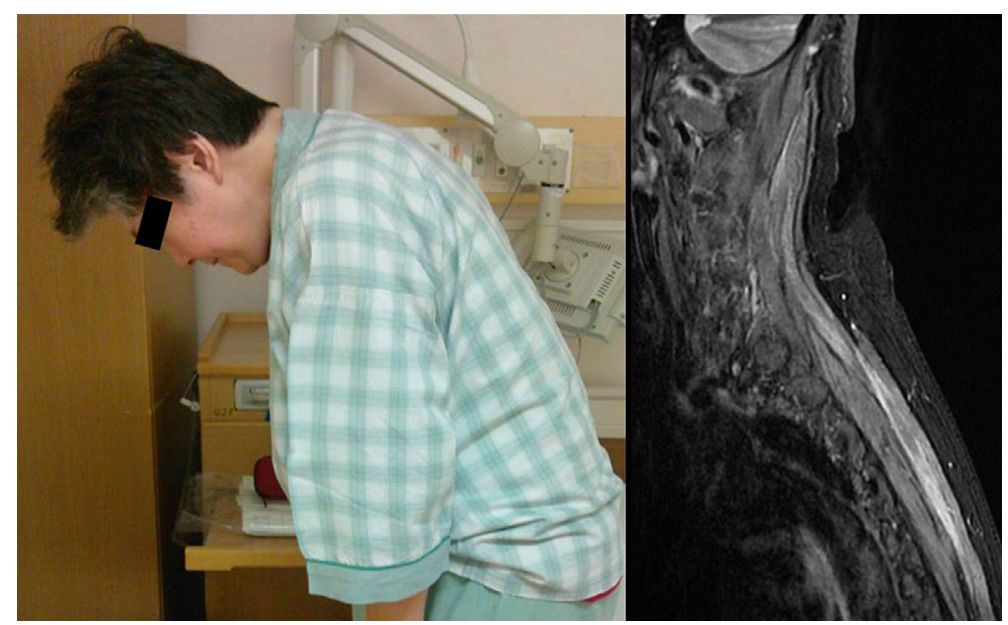

Picture 1.

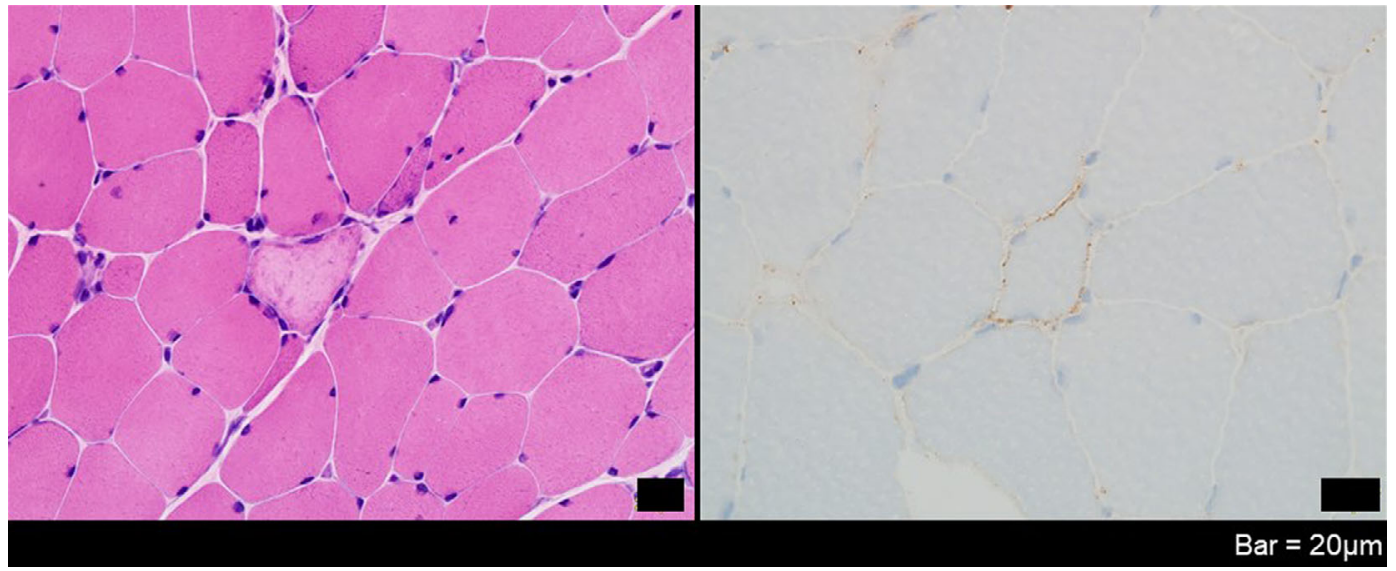

Picture 2.

${ }^{1}$ Department of Neurology, Aomori Prefectural Central Hospital, Japan and ${ }^{2}$ Department of Neuromuscular Research, National Institute of Neuroscience, National Center of Neurology and Psychiatry (NCNP), Japan

Received: February 28, 2019; Accepted: June 10, 2019; Advance Publication by J-STAGE: July 22, 2019

Correspondence to Dr. Tatsuya Ueno, tatsuya_ueno@med.pref.aomori.jp 


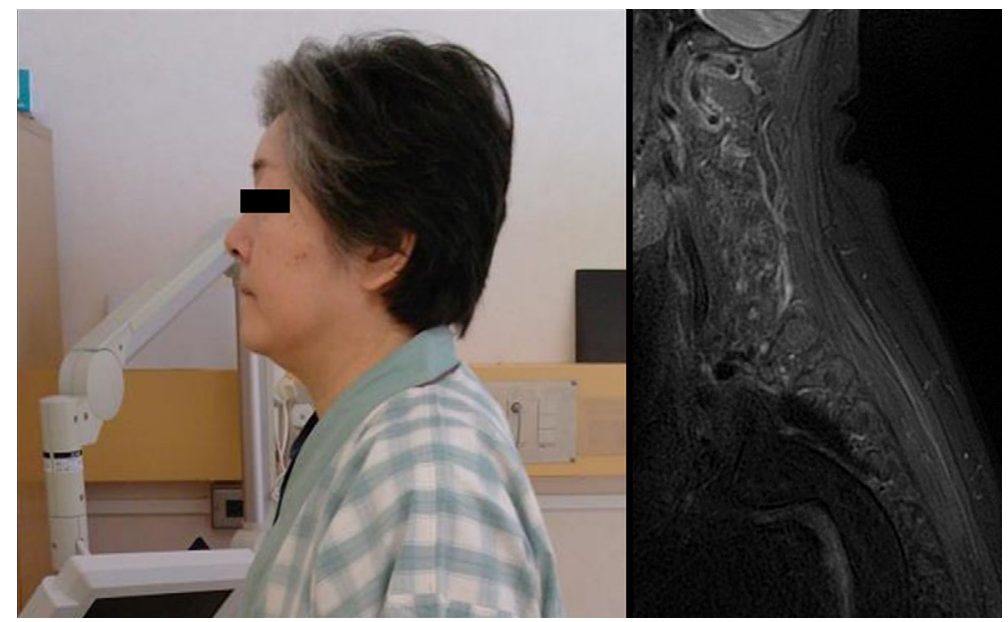

Picture 3.

The patient was a 60-year-old Japanese woman who presented with dropped head syndrome (DHS) and limb weakness (Picture 1). A neurological examination showed proximal dominant limb and truncal weakness. The neck extensor strength was weaker than the neck flexor strength. She had increased creatine kinase levels $(8,430 \mathrm{U} / \mathrm{L})$ and anti-signal recognition particle antibodies. Short-tau inversion recovery images showed paraspinal muscle hyperintensity (Picture 1). Hematoxylin-eosin staining of a left biceps muscle specimen showed mild-to-moderate fiber size variation, and necrotic and degenerating processes without mononuclear cell infiltration (Picture 2). Immunostaining for membrane attack complex was positive in the sarcolemma of necrotic fibers (Picture 2). The results of the histopathological evaluation were compatible with immune-mediated necrotizing myopathy (IMNM). DHS and the short-tau inversion recovery abnormality improved after intravenous methylprednisolone pulse therapy followed by treatment with oral prednisolone and tacrolimus (Picture 3).

DHS is characterized by severe neck extensor weakness and occurs in various neuromuscular disorders (1). Although neck weakness is found in IMNM, the association of DHS is unknown (2). Clinicians should consider IMNM if patients with DHS have hyper-creatine kinase-emia.

The patient provided her written informed consent for the publication of this report.

The authors state that they have no Conflict of Interest (COI).

\section{References}

1. Katz JS, Wolfe GI, Burns DK, Bryan WW, Fleckenstein JL, Barohn RJ. Isolated neck extensor myopathy: a common cause of dropped head syndrome. Neurology 46: 917-921, 1996.

2. Watanabe Y, Uruha A, Suzuki S, et al. Clinical features and prognosis in anti-SRP and anti-HMGCR necrotising myopathy. J Neurol Neurosurg Psychiatry 87: 1038-1044, 2016.

The Internal Medicine is an Open Access journal distributed under the Creative Commons Attribution-NonCommercial-NoDerivatives 4.0 International License. To view the details of this license, please visit (https://creativecommons.org/licenses/ by-nc-nd/4.0/).

(C) 2019 The Japanese Society of Internal Medicine Intern Med 58: 3343-3344, 2019 